\title{
The Middle Sample Based on Color Face Identification Feature Extraction and Automatic Identification
}

\author{
Ying Ouyang*, Yongjun Liu \\ Suzhou 215500 \\ *Corresponding Author: ouyy@ cslg.edu.cn
}

School of computer science and technology, Changshu Institute of Technology,

\begin{abstract}
Currently face recognition has made some success, algorithms are constantly being improved, this paper puts forward the sample was based on the middle sample what will consider existing deviation from the samples and it will affect samples, the average face recognition rate dropped to sample, therefore consider removing part of samples of the sample among affect outcomes. Combined with colored face feature identified and automatic identification, the improved algorithm will be applied to color facial identification. Finally, extensive experiments performed on the international and universal AR standard color face database verify the effectiveness of the proposed method.
\end{abstract}

Keywords:middle samples, color face recognition, discriminating characteristics, feature extraction, automatic identification

\section{Introduction}

In recent years, as a research subject with theoretical and application value, face recognition has being attached importance and attention to researchers. By this, varieties of recognition methods, one of which is Principal Component Analysis [1-4] (PCA), have emerged one after another. The traditional PCA method follows the following basic processing: by the $\mathrm{K}-\mathrm{L}$ [5-8] transformation, we can extract the major ingredient of human face to establish Eigen-face space, then project test images into this Eigen-face space to get a group of projection coefficients, finally identify through comparing the projection coefficients with that of every face image. However, in the algorithm we calculate the sample mean, according to which we can get the deviation matrix and then get the general scatter-difference[9], but because of the deviation of some samples, the sample mean is not so accurate, thereby the general scatter-difference if affected, and the recognition rate is decreased finally. To deal with this problem, this paper puts forward a method based on middle sample, which is also applied to gray-scale face image. It turns out the recognition rate is increased when color information is adopted comparing to that of recognition which just concern gray-scale image, therefore, more and more researchers begin to use the color information of face image to improve the performance of face recognition algorithm. But the basic idea of color face recognition is transforming color face image into gray-scale face image, then color face recognition is based on gray-scale image. So face recognition algorithm based on gray-scale image is still pervasive. This report applies the improved method to the identification of color face [10-11].

Starting from original color algebraic feature of images, analyzing the characteristics of the formation of color face image, the paper introduces how to extract principal component feature from the three components R, G, B of color face image and analyzed it [12-13], then experiments are tested by adopting the improved PCA. What's more, all of the results were validated through the international AR standard color face database.

\section{Fisher linear discriminant analysis}

\subsection{Basic ideal of FLDA}

PCA is the optimal orthogonal transformation, of which the basic idea is to get the direction projection of maximum general scatter-difference by covariance matrix of sample. The advantage of PCA lies that it eliminated the correction between the various components of sample vector. 
Eigen-face subspace it generates has the best representation of characteristics, but it didn't utilize category information of the training sample efficiently, therefore PCA is not the optimal at the sense of classification and identification. Fisher Linear Discriminant Analysis (FLDA) has compensated for the shortcoming. The basic idea of FDLA is to get a projection space in which the ration of dispersion of between-class and within-class is the maximum, namely reaching between-class dispersion maximum and within-class minimum. It means to make the distance between different classes as large as possible, and the distance within class as small as possible. In figure 1, hollow circles and solid circle represent the two different types of samples; the figure represents the distribution of the two types of samples in two different projection directions $\boldsymbol{W}_{1}$ and $\boldsymbol{W}_{2}$ after projection. As shown in figure.1, projection got in direction $\boldsymbol{W}_{1}$ can separate the two types of samples well, however, part of projection in direction $\boldsymbol{W}_{2}$ overlaps together, it is difficult to separate the two types of samples. Thereby, whether to quest optimum projection direction is important.
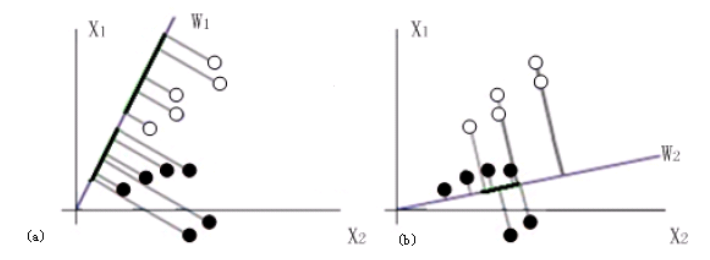

Figure. 1 sample projection distribution (a) meeting the

requirment of Fisher criterion (b) not meeting the

$$
\text { requirment of Fisher criterion }
$$

The classical linear discriminant analysis takes the fisher linear discriminant function, so that linear discriminant analysis is also called Fisher Linear Discriminant Analysis. The basis ideal of Fisher criterion lies in getting the optimum projection direction, on which the overlap of projections of the two types of samples is the minimum, so as to achieve the best classification results. Fisher criterion was first proposed by R.A .in 1936, and was applied to face recognition by Swets and Weng in 1996.

\subsection{Principles of FLDA and algorithm}

Training sample set of face image $\left\{\boldsymbol{x}_{1}, \boldsymbol{x}_{2}, \cdots, \boldsymbol{x}_{N}\right\}$,

$\boldsymbol{x}_{i}$ is $n$-dimensional vector. Training samples are divided

into $K$ classes, recorded as $C_{1}, C_{2}, \cdots, C_{K}$, and there are

$N_{i}$ training samples in class $C_{i}$. Then mean vector of all samples is defined as:

$\boldsymbol{m}=\frac{1}{N} \sum_{i=1}^{N} \boldsymbol{x}_{i}$

Mean vector of class $C_{i}$ is defined as:

$\boldsymbol{m}_{i}=\frac{1}{N_{i}} \sum_{\boldsymbol{x}_{i} \in C_{i}} \boldsymbol{x}_{i}$

The between-class scatter matrix is defined as:

$\boldsymbol{S}_{b}=\frac{1}{N} \sum_{i=1}^{K} N_{i}\left(\boldsymbol{m}_{i}-\boldsymbol{m}\right)\left(\boldsymbol{m}_{i}-\boldsymbol{m}\right)^{T}$

The within-class scatter matrix is defined as:

$\boldsymbol{S}_{w}=\frac{1}{N} \sum_{i=1}^{K} \sum_{x_{i} \in C_{i}}\left(\boldsymbol{x}_{i}-\boldsymbol{m}_{i}\right)\left(\boldsymbol{x}_{i}-\boldsymbol{m}_{i}\right)^{T}$

The total scatter matrix is defined as:

$\boldsymbol{S}_{t}=\boldsymbol{S}_{b}+\boldsymbol{S}_{w}=\frac{1}{N} \sum_{i=1}^{N}\left(\boldsymbol{x}_{i}-\boldsymbol{m}\right)\left(\boldsymbol{x}_{i}-\boldsymbol{m}\right)^{T}$

Obviously, $\boldsymbol{S}_{t}$ is the covariance matrix of all samples.

FLAD algorithm is to find the best projection direction $\boldsymbol{W}_{\text {opt }}$, which is the orthogonal eigenvector when the ratio of scatter matrix between and within class is the maximum, Fisher criterion function is defined as:

$$
J\left(\boldsymbol{W}_{\text {opt }}\right)=\arg \max \frac{\left|\boldsymbol{W}^{T} \boldsymbol{S}_{b} \boldsymbol{W}\right|}{\left|\boldsymbol{W}^{T} \boldsymbol{S}_{w} \boldsymbol{W}\right|}
$$

$\boldsymbol{W}_{\text {opt }}$ is the solution meets the following equation:

$\boldsymbol{S}_{b} \boldsymbol{W}_{i}=\lambda_{i} \boldsymbol{S}_{w} \boldsymbol{W}_{i}, \quad i=1,2 ; \cdot m$ 
It corresponds to eigenvector that corresponds to the first $m$ larger eigenvalue $\lambda_{i}$ of $\boldsymbol{S}_{w}^{-1} \boldsymbol{S}_{b}$.

However, we often encounter such a problem: scatter matrix within class is a singular, so that we can't get eigenvalue of $\boldsymbol{S}_{w}^{-1} \boldsymbol{S}_{b}$ directly. It is evoked that number of training samples is often less than the number of pixies of the samples. This is so-called small sample problem.

\section{Our method}

Because there are interference samples far away from all the samples, the article proposed a following new viewpoint: remove interference samples and seek middle sample, we can ensure that samples acquired not be influenced by some samples. Suppose there is a sample set $\left\{\mathcal{X}_{1}, \mathcal{X}_{2}, \ldots \mathcal{X}_{N}\right\}$, of which $\boldsymbol{X}_{i}$ is a dimensional vector, while training samples are divided into $\mathrm{k}$ classes, recorded as $C_{1}, C_{2}, \ldots, C_{k}$, and there are $M$ training samples in each class. As shown in figure.2, each column represents a sample. Firstly sorting all the samples in sample set $\left\{\mathcal{X}_{1}, \mathcal{X}_{2}, \ldots \mathcal{X}_{N}\right\}$ according to their pixel value, in other words, sorting pixel value in the red box of the figure to form a new sample set $\left\{x_{1}{ }^{\prime}, x_{2}{ }^{\prime}, \ldots x_{N}{ }^{\prime}\right\}$, then we fetch the middle sample of the new sample set as mean vector $\bar{x}_{a}$ of all the samples.

$$
\left[\begin{array}{cccc}
x_{1}(1) & x_{2}(1) & \cdots & x_{N}(1) \\
x_{1}(2) & x_{2}(2) & \cdots & x_{N}(2) \\
\cdots & \cdots & \cdots & \cdots \\
x_{1}(n) & x_{2}(n) & \cdots & x_{N}(n)
\end{array}\right]
$$

Figure.2 Matrix representation of all samples

Under the new assumptions, new overall mean vector $\bar{x}^{a b}$ and mean vector of every class $\overline{\boldsymbol{X}}^{a b}$ are substituted to construct new scatter matrix by $\bar{x}^{a b}$ and $\bar{x}_{i}^{a b}$.

The new between-class scatter matrix is defined as:

$$
\boldsymbol{S}_{b}^{a b}=\frac{1}{N} \sum_{i=1}^{K} N_{i}\left(\boldsymbol{x}_{i}^{a b}-\overline{\boldsymbol{x}}^{a b}\right)\left(\boldsymbol{x}_{i}^{a b}-\overline{\boldsymbol{x}}^{a b}\right)^{T}
$$

The new within-class scatter matrix is defined as:

$$
\boldsymbol{S}_{w}^{a b}=\frac{1}{N} \sum_{i=1}^{K} \sum_{x_{i} \in C_{i}}\left(\boldsymbol{x}_{i}-\overline{\boldsymbol{x}}_{i}^{a b}\right)\left(\boldsymbol{x}_{i}-\overline{\boldsymbol{x}}_{i}^{a b}\right)^{T}
$$

The total scatter matrix is defined as:

$$
S_{t}^{a b}=S_{b}^{a b}+S_{w}^{a b}=\frac{1}{N} \sum_{i=1}^{N}\left(\boldsymbol{x}_{i}-\overline{\boldsymbol{x}}^{a b}\right)\left(\boldsymbol{x}_{i}-\overline{\boldsymbol{x}}^{a b}\right)^{T}
$$

Plug the obtained scatter matrix into Fisher criterion of the Eq.11 and maximum scatter difference discriminant criterion of the Eq.12

$$
\begin{aligned}
& J\left(\boldsymbol{W}_{\text {opt }}\right)=\arg \max \frac{\left|\boldsymbol{W}^{T} \boldsymbol{S}_{b} \boldsymbol{W}\right|}{\left|\boldsymbol{W}^{T} \boldsymbol{S}_{w} \boldsymbol{W}\right|} \\
& \left\{\begin{array}{l}
J_{s}(\boldsymbol{\varphi})=\varphi^{T} \boldsymbol{S}_{b} \boldsymbol{\varphi}-\boldsymbol{\varphi}^{T} \boldsymbol{S}_{w} \boldsymbol{\varphi}=\boldsymbol{\varphi}^{T}\left(\boldsymbol{S}_{b}-\boldsymbol{S}_{w}\right) \boldsymbol{\varphi} \\
\boldsymbol{\varphi}^{T} \boldsymbol{\varphi}=1
\end{array}\right.
\end{aligned}
$$

We have to solve new projection direction for face identification feature extraction, measure similarity and classify to apply the new method to color facial identification.

Color face identification feature extraction and automatic identification is to find "feature space" 
$U_{R}, U_{G}, U_{B}$ of three color spaces by steps to create eigenface space based on principal component method, next, project the color image, any face image after centralization can be projected into eigenface subspace and get a group of coordinate coefficients:

$$
\tilde{x}^{i}=U^{T}{ }^{-i}
$$

Projections of three color spaces of color image can be expressed as follows:

\section{$\mathrm{R}$ component:}

$$
\tilde{x}_{R}^{i}=U_{R}{ }^{T} \stackrel{-i}{x_{R}}
$$

$\mathrm{G}$ component:

$$
\tilde{x}_{G}^{i}=U_{G}^{T}{ }^{-i} x_{G}
$$

B component:

$$
\tilde{x}_{B}^{i}=U_{B}{ }^{T}{ }^{-i}{ }_{B}
$$

Finally, input the selected discriminating characteristics into classifier for classification, the most classic classifier is the nearest neighbor classifier or minimum distance classifier.

\section{Experimental results and analysis}

In order to verify the validity of the proposed algorithm, the experimental is based on a large AR face image database. The database is made up of 26 face images per person which were taken in different periods, light, gesture, facial expressions and occlusion conditions from 120 persons. The 26 images were taken in two periods, the former 13 images were taken in one period, and the latter 13 images were taken in another period, there is a difference of 14 days before and after. The experiment only investigated unobstructed images; take images from the former 60 persons as the sample set. The experiment used standardized face images with a resolution of $50 \times 40$. Images shown in figure. 31 are 14 color images of someone after standardization.

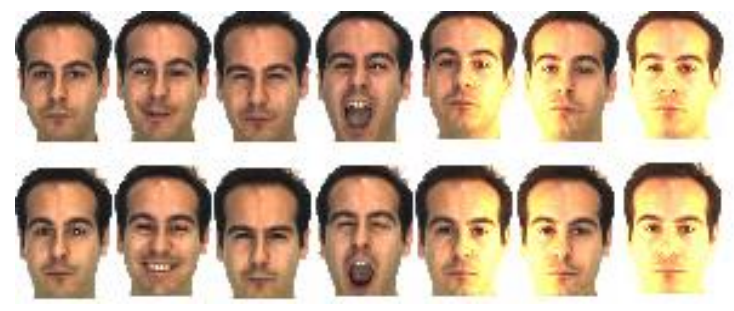

Figure.3 14 images of AR face image database

The experiment adopts linear discriminant analysis to establish within-class scatter matrix and between-class scatter matrix. As a result, the dimensions will be high, and obviously such a high number of dimensions are unrealistic to run on computers. Therefore the experiment first reduced the dimensionality of sample's three RGB components by principal component analysis, then used Fisher criterion and maximum scatter difference criterion for linear discriminant analysis. As shown in table.1 and table.2, they extracted feature on projection axis 50-59, they are comparison table of recognition rate and comparison table of time used using the nearest neighbor classifier and minimum distance classifier.

\begin{tabular}{|c|c|c|c|c|}
\hline \multirow{2}{*}{$\begin{array}{l}\text { Numbe } \\
\text { r of } \\
\text { projecti } \\
\text { on axes }\end{array}$} & \multicolumn{2}{|c|}{$\begin{array}{c}\text { Improved Fisher } \\
\text { criterion }\end{array}$} & \multicolumn{2}{|c|}{$\begin{array}{l}\text { Improved maximum } \\
\text { scatter difference } \\
\text { criterion }\end{array}$} \\
\hline & MD & $1 \mathrm{NN}$ & MD & $1 \mathrm{NN}$ \\
\hline 50 & $85.0 \%$ & $86.5 \%$ & $85.5 \%$ & $86.5 \%$ \\
\hline 51 & $85.5 \%$ & $87.0 \%$ & $85.5 \%$ & $87.5 \%$ \\
\hline 52 & $86.5 \%$ & $88.5 \%$ & $85.0 \%$ & $89.0 \%$ \\
\hline 53 & $86.5 \%$ & $88.0 \%$ & $85.0 \%$ & $88.5 \%$ \\
\hline 54 & $88.5 \%$ & $88.0 \%$ & $85.0 \%$ & $88.5 \%$ \\
\hline 55 & $88.5 \%$ & $89.0 \%$ & $85.0 \%$ & $89.0 \%$ \\
\hline 56 & $88.5 \%$ & $89.0 \%$ & $85.5 \%$ & $89.0 \%$ \\
\hline 57 & $89.0 \%$ & $89.5 \%$ & $86.0 \%$ & $89.0 \%$ \\
\hline 58 & $89.0 \%$ & $90.0 \%$ & $86.0 \%$ & $89.0 \%$ \\
\hline 59 & $89.5 \%$ & $91.5 \%$ & $85.5 \%$ & $89.0 \%$ \\
\hline
\end{tabular}

Table.1 Comparison table of recognition rate using two kind of classifier by two improved discriminant analysis methods which use $\bar{x}_{a}$

Table. 2 Comparison table of recognition rate from two kind of classifier after two improved discriminant analysis methods by utilizing $\bar{x}_{b}$ 


\begin{tabular}{|c|c|c|c|c|}
\hline \multirow{2}{*}{$\begin{array}{l}\text { NO. } \\
\text { projection } \\
\text { axes }\end{array}$} & \multicolumn{2}{|c|}{$\begin{array}{c}\text { Improved Fisher } \\
\text { criterion }\end{array}$} & \multicolumn{2}{|c|}{$\begin{array}{l}\text { Improved maximum } \\
\text { scatter difference } \\
\text { criterion }\end{array}$} \\
\hline & MD & $1 \mathrm{NN}$ & MD & $1 \mathrm{NN}$ \\
\hline 50 & $86.0 \%$ & $86.5 \%$ & $86.5 \%$ & $86.5 \%$ \\
\hline 51 & $86.0 \%$ & $87.5 \%$ & $86.5 \%$ & $87.0 \%$ \\
\hline 52 & $87.5 \%$ & $89.0 \%$ & $88.0 \%$ & $89.5 \%$ \\
\hline 53 & $87.5 \%$ & $88.0 \%$ & $88.0 \%$ & $88.5 \%$ \\
\hline 54 & $88.5 \%$ & $88.5 \%$ & $88.0 \%$ & $88.0 \%$ \\
\hline 55 & $89.5 \%$ & $90.0 \%$ & $89.0 \%$ & $89.5 \%$ \\
\hline 56 & $89.5 \%$ & $90.5 \%$ & $89.5 \%$ & $90.0 \%$ \\
\hline 57 & $89.0 \%$ & $91.5 \%$ & $90.0 \%$ & $91.0 \%$ \\
\hline 58 & $90.0 \%$ & $92.0 \%$ & $90.0 \%$ & $91.0 \%$ \\
\hline 59 & $90.5 \%$ & $91.5 \%$ & $91.5 \%$ & $90.0 \%$ \\
\hline
\end{tabular}

Table. 3 comparison table of time used under optimal recognition rate when using the nearest neighbor classifier

by two improved discriminant analysis methods

\begin{tabular}{|c|c|c|c|}
\hline $\begin{array}{c}\text { Identification } \\
\text { criteria }\end{array}$ & $\begin{array}{c}\text { Time for feature } \\
\text { extraction(s) }\end{array}$ & $\begin{array}{c}\text { Time for } \\
\text { classification(s) }\end{array}$ & $\begin{array}{c}\text { Total } \\
\text { time(s) }\end{array}$ \\
\hline $\begin{array}{c}\text { Maximum scatter } \\
\text { difference criterion } \\
(47)\end{array}$ & 143.57 & 3.34 & 146.91 \\
\hline $\begin{array}{c}\text { Fisher criterion } \\
(49)\end{array}$ & 177.43 & 3.46 & 180.89 \\
\hline
\end{tabular}

Note: Numbers in brackets is the number of select projection axis

As shown in table1.and table.2, the recognition rate has been increased through Fisher criterion and improved maximum scatter difference criterion improved in the first and second program especially the second program duo to the two programs removed the effect of some interference samples. As shown in table.3, because of the addition of average sample problems, time for feature extraction is significantly increased, especially in the second program.

\section{Conclusion}

The paper puts forward a kind of face recognition based on the middle sample, which gets the average of some samples rather than gets average of all samples. This method excludes the influence of the interference samples on experiments and increased the recognition rate significantly. As researches nowadays are mainly based on the gray-scale ones, this article applies improved methods to color face identification. Color face recognition is to transfer the color images into gray images, then use the extraction idea of the latter. Finally, through combination of the experiment and the new ideas, we find that the method is feasible.

In the end, large numbers of experiments based on international general AR standard color face database have adequately proved the validation of the method for processing color face image identification.

\section{References}

(1)Robert W. Frischholz, Ulrich Diecklmann. BioID, "A Multimodal Biometric Identification System", IEEE Computer,vol.33,no.2,pp.64-68,2000.

(2)JIANG Qing-Ling, "Edge Detection for Color Image Based on CNN", Journal of AISS, AISS(Advances in Information Sciences and Service Sciences), Vol.3, No.10, pp. 61-69, 2011.

(3)Turk Matthew, Pentland Alex, "Face recognition using Eigenfaces", Proc. IEEE Conf. On Computer Vision and Pattern Recognition, pp.586-591, 1991.

(4)Pentland Alex, "Looking at people: sensing for ubiquitous and wearable computing", IEEE Trans. Pattern Anal.

Machine Intell,vol.22,no.1,pp.107-119,2000.

(5)Maxim A, Grudin, "On internal representations in face recognition systems", Journal of Pattern Recognition, vol.33, no.7, pp.1161-1177,2000.

(6)Shih P, Liu C, "Comparative assessment of content based face image retrieval in different color spaces", Journal of Pattern Recognition , vol 19,no.7,pp.873-893,2005.

(7)Creed J, Abbott A. Color face recognition by hyper complex gabor analysis[C], Proceedings of the 7th International Conference on Automatic Face..

(8)Yang Hai, Wei He, Xin Liu, Lei Fan, "An Extracting Algorithm for Classification Rule based on Frequent Concept Set", Journal of AISS, AISS(Advances in Information Sciences and Service Sciences), Vol. 3, No. 1, pp. 41-46, 2011.

(9)Chen L F, Mark-Liao, et at. "A new LDA-based face recognition system which can solve the small sample size problem", Pattern Recognition, no.33, pp.1713-1726, 2000.

(10)Turk M and Pentland A, "Face Recognition Using Eigenfaces", In Proceeding of the IEEE Computer 
Society Conference on Computer Vision and Pattern Recognition, pp.586-591,1994.

(11)Pentland A et al. View-based Modular Eigenspaces for Face Recognition. Proc. IEEE Conf. on CVPR, 84-91, 1994.

(12)Yu H, Yang J, "A Direct LDA Algorithm for High-Dimensional Data With Application to Face Recognition", Journal of Pattern Recognition, 2001, 34(10):2067-2070.

(13) Liu Yong-jun, Chen Cai-kou, "Differential Space-based Maximum Scatter Difference Discriminant Analysis and Face Recognition" , Journal of Computer Applications, 2006. 